\title{
Elevated expression of GNAS promotes breast cancer cell proliferation and migration via the $\mathrm{PI} 3 \mathrm{~K} / \mathrm{AKT} / \mathrm{Snail}$ /E-cadherin axis
}

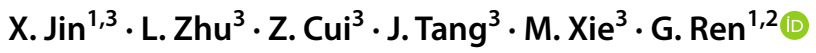

Received: 3 September 2018 / Accepted: 14 January 2019 / Published online: 14 February 2019

(c) The Author(s) 2019

\begin{abstract}
Purpose Although it has been well established that $\mathrm{G}$ protein plays pivotal roles in physiologic or pathologic conditions, including cancer formation, its role in breast cancer, especially specific subunits, remains largely unknown. Our work aimed to evaluate the correlation of the G protein alpha subunit (GNAS) with breast cancer and to investigate the underlying molecular mechanism.

Methods The expression of GNAS was determined by breast tumor tissue microarray of 150 patients with complete followup information. The correlation between GNAS expression and clinical features was assessed. CCK8, EdU incorporation, flow cytometry, wound healing, transwell, western blot and tumor formation assays were carried out in nude mice to study the biological function of GNAS and the underlying molecular mechanism in breast cancer by silencing GNAS using a specific siRNA.

Results High GNAS expression showed a close correlation with a reduced overall survival $(p=0.021)$, frequent distal metastasis $(p=0.026)$, advanced clinical stage $(p=0.001)$, stronger cell proliferation (ki67 $7^{+}$positive cell rate, $p=0.0351$ ) and enhanced cancer cell migration, which was further confirmed by in vitro and in vivo assays and might be dependent on the PI3K/AKT/Snail1/E-cadherin axis.

Conclusion The data suggested that GNAS promoted breast cancer cell proliferation and migration (EMT) through the $\mathrm{PI} 3 \mathrm{~K} / \mathrm{AKT} / \mathrm{Snail1} / \mathrm{E}$-cadherin signaling pathway. These findings also indicate that GNAS can serve as a potential prognostic indicator and novel therapeutic target in breast cancer.
\end{abstract}

Keywords GNAS $\cdot$ Breast cancer $\cdot$ PI3K/AKT signaling $\cdot$ Cell proliferation $\cdot$ Cell migration $\cdot$ Metastasis

\section{Introduction}

Breast cancer is the most frequently diagnosed cancer and the leading cause of cancer-related mortality in women worldwide [1]. Uncontrolled proliferative and high metastatic abilities are the most distinctive features. Although

\section{G. Ren}

guoshengren65@163.com

1 Department of Endocrine and Breast Surgery, The First Affiliated Hospital of Chongqing Medical University, No. 1, Youyi Road, Yuanjiagang, Yuzhong District, Chongqing 400016, China

2 Chongqing Key Laboratory of Molecular Oncology and Epigenetics, The First Affiliated Hospital of Chongqing Medical University, Chongqing, China

3 Department of Breast Surgery, The First People's Hospital of Yibin, Yibin, Sichuan, China early detection of primary tumors may allow effective treatment, metastatic cases are largely disastrous, incurable and represent the ultimate cause of mortality in breast cancer patients. It is estimated that approximately $6 \%$ of patients already have metastatic disease at the time of diagnosis, while approximately $20-50 \%$ of patients who are initially diagnosed with early stage breast cancer will eventually develop metastasis [2]. Although progress in breast cancerrelated basic research has recently been achieved, exploration of the critical drivers of uncontrolled proliferation and metastasis and investigations of the underlying mechanism are still desperately needed.

G protein-coupled receptors (GPCRs), possessing seventransmembrane domains, play pivotal roles in physiological or pathological processes by modulating downstream signaling pathways [3, 4]; the dysregulation of GPCR signaling members has been recognized as a hallmark of cancer [5]. Abnormal expression of specific GPCRs induces continual 
uncontrolled cell proliferation, triggers intracellular signal transduction and ultimately leads to the growth of cancer cells, inducing angiogenesis and metastasis, and approximately $25 \%$ of marketed pharmaceuticals target human GPCRs or their signaling pathways [6]. However, it should be mentioned that signal transduction by GPCRs is mainly dependent on $\mathrm{G}$ proteins.

There are two classes of $\mathrm{G}$ proteins: the first class functions as a monomeric small GTPase, while the second class, called heterotrimeric $\mathrm{G}$ proteins, consists of $\alpha, \beta$, and $\gamma$ subunits and functions as a molecular switch [7]. When combined with a ligand, GDP is replaced by GTP and is released from the Gs $\alpha$ subunit (GNAS), the stimulatory $\alpha$ subunit of the $\mathrm{G}$ protein, followed by the dissociation of Gs $\alpha$ from the $\beta, \gamma$ units. Gs $\alpha$ activates the cAMP-dependent pathway via stimulation of cAMP production from ATP. cAMP then acts as a second messenger that interacts with and activates protein kinase A (PKA), which phosphorylates countless downstream targets that are involved in a number of pathways and evokes downstream signaling cascades [8,9].

Although, accumulating evidence has demonstrated that GPCRs, such as GPCR81, PAR1, GPR110, GPR19 and especially G protein-coupled estrogen receptor (GPER), are tightly associated with the malignant transformation of mammary cells, the detailed functions of GNAS in breast cancer and their correlation with clinical features are still missing [10-12]. In this study, we focused on exploring the correlation of GNAS with breast cancer and investigating the underlying molecular mechanism.

\section{Methods}

\section{Patients and sample preparation}

We studied 150 breast tumor tissues from a cohort of 217 patients diagnosed with breast cancer who underwent tumor removal at the First People's Hospital of Yibin between 2006 and 2009. Overall survival (OS) was defined as the time between initial surgery and death. We prepared tissue microarray (TMA) cores (1.5 mm diameter) from formalin-fixed, paraffin-embedded samples. IHC staining was performed on all of the TMA slides, and the results were interpreted by two pathologists using a blinded method. GNAS staining were scored according to the cytoplasmic staining intensity: $0-2$ indicated low staining and 3-4 high staining. The mean score was the final score. Classical core clinical characteristics, such as the WHO grade, clinical stage, tumor size, nodal status, distal metastasis, ER stage, PR stage and Her-2 stage, were included to analyze the correlation of GNAS with breast cancer. Moreover, univariate and multivariate analyses of different prognostic variables of GNAS with overall survival were performed. Approval for this study was granted by the Ethics Committee of the First People's Hospital of Yibin.

\section{Cell culture}

The human breast cancer cell lines, including MCF-7, MDAMB-231, MDA-MB-468, BT-474 and SK-BR-3, were purchased from the ATCC. The cells were cultured in RMPI Medium 1640 supplemented with $10 \%$ fetal bovine serum (FBS), $1 \%$ penicillin and $1 \%$ streptomycin at $37{ }^{\circ} \mathrm{C}$ in a $5 \%$ $\mathrm{CO} 2$ humidified incubator. To specifically inhibit PI3Ks, $0.2 \mu \mathrm{M}$ NVP-BKM120 hydrochloride (BKM120) was used.

\section{Transfection}

For transfection, cells were washed with serum-free medium once and then incubated with serum-free medium for $4 \mathrm{~h}$. The siGNAS (5'-TGCATGTTAATGGGTTTAA- ${ }^{\prime}$ ' and 5'-ACTACTGCTACCCTCATTT-3'), siControl (RiBio, Guangzhou, China) and lipofectamine 2000 (Invitrogen, CA, USA) were separately mixed with $500 \mu$ l of Opti-MEM I Reduced Serum Medium (Gibco, Grand Island, NY) for $5 \mathrm{~min}$. Then, the two mixtures were combined and incubated at room temperature for $20 \mathrm{~min}$. The lipofectamine: siRNA mixture was added to the cells and incubated at $37{ }^{\circ} \mathrm{C}$ for $6 \mathrm{~h}$. Subsequently, fresh medium containing $10 \%$ FBS was added, and the cells were maintained in culture until the following experiments.

\section{Proliferation and cell viability assay}

CCK8 (Sigma-Aldrich Co., St Louis, MO, USA) and EdU incorporation (RiboBio, Guangzhou, China) assays were carried out to evaluate cell viability and proliferation according to the manufacturers' instructions.

\section{Cell-cycle and apoptosis assay}

Cell cycle and apoptosis were measured using the Cell Cycle and Apoptosis Analysis Kit according to the manufacturer's instruction (Beyotime Biotechnology, Shanghai, China). The results were analyzed with FlowJo software.

\section{Wound healing assay}

Approximately $2 \times 10^{5}$ cells were plated in 6-well plates after the different treatments. A linear scratch was generated on the cell monolayer with a sterile pipette. Photomicrographs of live cells were obtained at $40 \times$ magnification, and the distance migrated was observed after $24 \mathrm{~h}$ or $48 \mathrm{~h}$. The remaining wound area was measured using ImageJ software. 


\section{Matrigel invasion assay}

The Matrigel invasion assay was performed in 24-well transwell culture plates. Cells were resuspended and then seeded in 24-well transwell plates containing FBS-free medium in the upper chamber and complete growth medium supplemented with $10 \%$ FBS in the lower chamber for $24 \mathrm{~h}$ at $37{ }^{\circ} \mathrm{C}$. Noninvading cells were removed from the upper surfaces of the invasion membranes, and the cells on the lower surface were stained with hematoxylin. The average number of cells per field was determined by counting the cells in six random fields per well. Cells were counted in four separate fields in three independent experiments.

\section{Soft agar colony formation}

A 6-well plate was coated with a 1:1 ratio of $1.2 \%$ agarose and $2 \times$ complete phenol red-free RIPM1640, and it was solidified for $30 \mathrm{~min}$. The top portion was prepared with $0.6 \%$ agarose and $2 \times$ medium with cells were plated at a density of 3000 cells $/ \mathrm{ml}$; a total of 1000 cells were used. Images were photographed after culturing for 14 days. Colonies were counted and statistically analyzed. Assays were performed three times using triplicate wells.

\section{Western blot}

Cells and tissues were lysed with RIPA buffer according to the manufacturer's instructions (Beyotime Biotechnology, Shanghai, China). Proteins were separated with $12 \%$ SDS-PAGE gels and transferred to PVDF membranes. Membranes were blocked with PBS containing $0.05 \%$ tween and $5 \%$ nonfat milk and probed with antibodies against GNAS, p-PKA, p85 $\alpha$, AKT, p-AKT, vimentin, E-cadherin, snail 1, slug, Cyclin D1, CDK4 and GAPDH (Huabio, Hangzhou, China). These antibodies were purchased from Abcam or CST if not mentioned. Signal intensities were quantified and normalized to the GAPDH intensity using ImageJ.

\section{Orthotopic nude mouse model and treatment}

BALB/c nude mice aged 4-6 weeks were purchased from the Animal Center at the Cancer Institute at the Chinese Academy of Medical Science (Beijing, China). Next, $1 \times 10^{6}$ MDA-MB-231 cells transfected with siGNAS or siControl were subcutaneously injected into the abdomen of each nude mouse. In addition, cholesterol-modified siGNAS or control siRNA (RiboBio, $5 \mathrm{nmol} / \mathrm{kg}$ ) dissolved in saline buffer were intratumorally injected every 3 days for 5 weeks. The tumors were measured weekly and harvested 5 weeks later.

\section{Statistical analysis}

Associations between the expression of GNAS and clinical variables as well as breast cancer were assessed using $t$ tests. The Kaplan-Meier method was used to estimate disease-free survival (DFS) and overall survival (OS), and the log-rank test was used to compare survival between two strata. The significance of different prognostic variables of GNAS for OS was analyzed in univariate and multivariate analyses. All tests were two-sided, and $p<0.05$ was considered statistically significant. All statistical analyses were performed using the SPSS version 19.0 software program (SPSS Inc., Chicago, USA).

\section{Results}

\section{Association between the expression of GNAS and survival and metastasis}

To identify the function of GNAS in breast cancer, we first examined the expression of GNAS in 150 breast cancer TMA samples with complete follow-up information. As a housekeeping protein, all the clinical samples were GNAS positive, and finally 97 samples were identified as the high expression and the other 53 as the low expression subtype (Fig. 1a). Western blot analysis revealed an elevated expression of GNAS in tumor tissues (Fig. 1b). The association of GNAS expression and the baseline clinical characteristics is presented in Tables 1 and 2. The impact of the clinicopathologic characteristics and prognostic factors was calculated by Kaplan-Meier analysis, and survival curves were delineated by the log-rank test. The results showed that high expression of GNAS was more likely to be observed in patient with distal metastasis $(p=0.026)$, a more advanced clinical stage $(p=0.001)$, and poor survival ( $p=0.021$, Fig. 1c). Moreover, we also examined ki-67 expression in GNAS -high and -low samples. The representative images and statistical results revealed that samples with high GNAS expression displayed more active proliferative activity ( $p=0.035$, Fig. 1 d).

\section{GNAS is crucial for breast cancer cell proliferation}

To further examine whether GNAS is important for the proliferation of breast cancer cells, we firstly evaluated the expression of GNAS in five different breast cancer cell lines (Fig. 2a). According to the results, the MDA-MB-231 cell line possessed the highest GNAS expression, while there was no obvious difference in MCF-7, BT-474, SK-BR-3 and MDA-MB-468. Therefore, MDA-MB-231 and MCF-7 were chosen for further study. To evaluate the role of GNAS in cell proliferation, specific siRNAs targeting GNAS were 

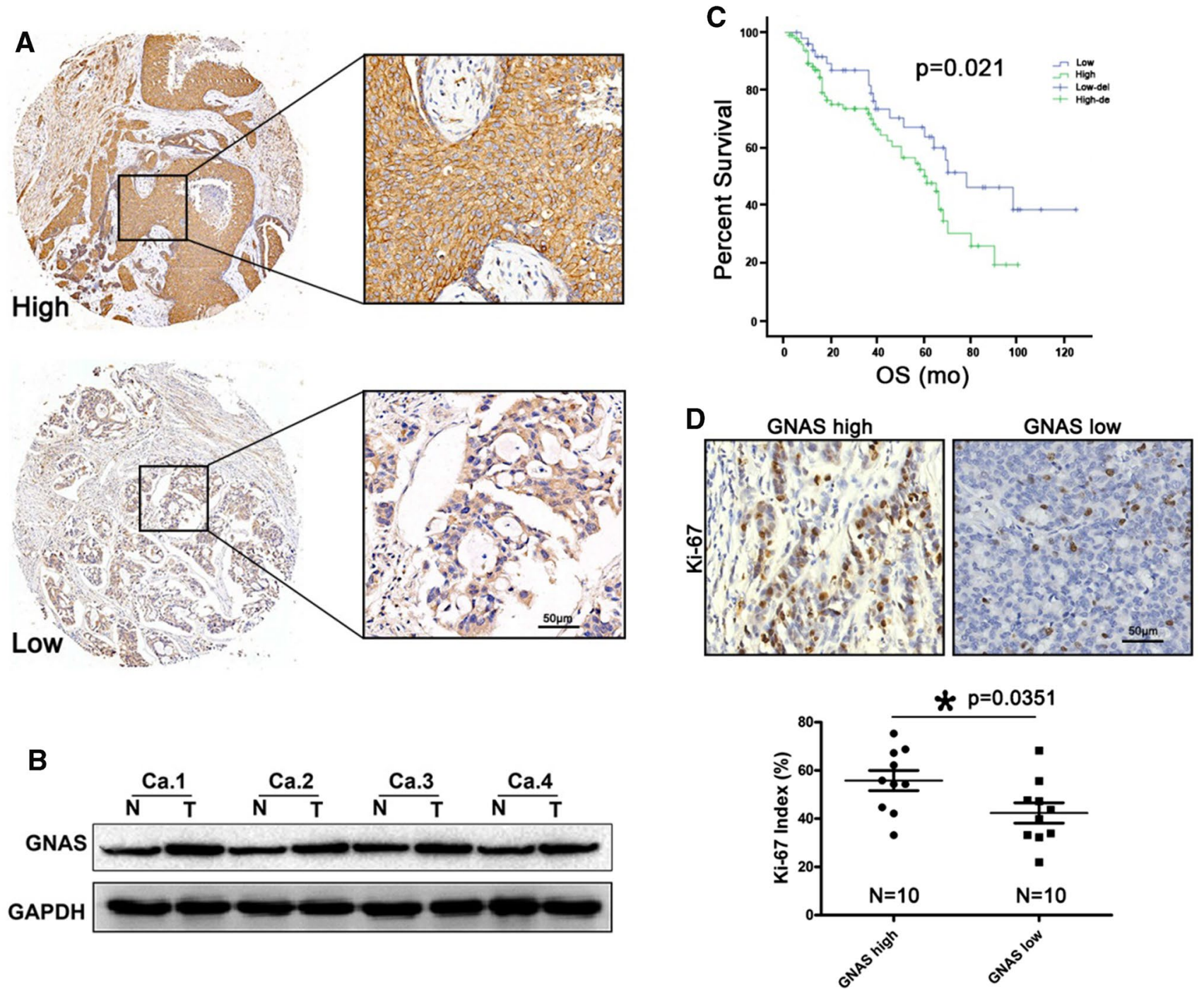

Fig. 1 The expression of GNAS and its relevance to the survival time and Ki-67 index in breast cancer. Representative figures with high or low GNAS expression, scale bar $=50 \mu \mathrm{m}(\mathbf{a})$; confirmation of the elevated expression of GNAS in tumor tissues compared with adja-

used, and both siRNAs dramatically decreased the protein level of GNAS after $72 \mathrm{~h}$ of transfection (Fig. 2b). The CCK8 assay results demonstrated that knockdown of GNAS markedly inhibited MCF-7 cell viability (Fig. 2c). Additionally, as shown in Fig. 2d, EdU incorporation assay revealed a reduced proliferation rate of MDA-MB-231 cells in the siGNAS-treated group $(70.883 \pm 11.37 \%$ vs $45.85 \pm 8.68 \%)$.

Furthermore, siGNAS decreased the percentage of MDAMB-231 cells in S phase and MDA-MB-231 cell proliferation rate. An increase in G1 phase was observed, which indicated that the cell cycle was arrested in G1 phase, which was further confirmed by the reduced expression of the G1-S transition marker Cyclin D1 and CDK4 (Fig. 3a, b). In contrast, no significant differences in apoptosis were observed cent tissues. $N$ nontumor or adjacent tumor tissue, $T$ tumor tissue (b); correlation of GNAS expression with the survival time of breast cancer patients (c); high expression of GNAS was associated with a high $\mathrm{Ki}-67$ index, scale bar $=50 \mu \mathrm{m}(\mathbf{d})$

in siGNAS- and siControl-treated cells (Fig. 3C). To obtain further evidence that GNAS facilitated the proliferation of breast cancer cell lines, a colony formation assay was performed. As shown in Fig. 3d, the number of colonies was lower in the siGNAS-treated group than the control group. The colony-forming ability of MDA-MB-231 and MCF-7 cells was significantly inhibited by $49 \%$ and $71 \%$, respectively ( $p<0.01$, Fig. $3 d$ ).

\section{Knockdown of GNAS impairs the motility and migration ability of breast cancer cells}

As a critical component of $\mathrm{G}$ protein, GNAS plays pivotal role in the process of intracellular and extracellular signal 
Table 1 The correlations of GNAS with clinicopathological features of breast cancer patients

\begin{tabular}{|c|c|c|c|c|}
\hline $\begin{array}{l}\text { Pathological } \\
\text { variables }\end{array}$ & Cases 150 & $\begin{array}{l}\text { High GNAS } \\
\text { expression } 97\end{array}$ & $\begin{array}{l}\text { Low GNAS } \\
\text { expres- } \\
\text { sion } 53\end{array}$ & $p$ value \\
\hline \multicolumn{5}{|l|}{ WHO grade } \\
\hline I & 52 & 21 & 31 & \multirow[t]{3}{*}{0.583} \\
\hline II & 44 & 29 & 15 & \\
\hline III & 54 & 47 & 7 & \\
\hline \multicolumn{5}{|c|}{ Tumor size $(\mathrm{cm})$} \\
\hline$<5$ & 74 & 43 & 31 & \multirow[t]{2}{*}{0.124} \\
\hline$\geq 5$ & 76 & 54 & 22 & \\
\hline \multicolumn{5}{|l|}{ Nodal status } \\
\hline N0-1 & 73 & 48 & 25 & \multirow[t]{2}{*}{0.865} \\
\hline $\mathrm{N} 2-3$ & 77 & 49 & 28 & \\
\hline \multicolumn{5}{|c|}{ Distal metastasis } \\
\hline Presence & 70 & 52 & 18 & \multirow[t]{2}{*}{$0.026^{*}$} \\
\hline Absence & 80 & 45 & 35 & \\
\hline \multicolumn{5}{|l|}{ Clinical stage } \\
\hline I-II & 63 & 31 & 32 & \multirow[t]{2}{*}{$0.001 * *$} \\
\hline III-IV & 87 & 66 & 21 & \\
\hline \multicolumn{5}{|l|}{ ER status } \\
\hline Positive & 78 & 51 & 27 & \multirow[t]{2}{*}{0.866} \\
\hline Negative & 72 & 46 & 26 & \\
\hline \multicolumn{5}{|l|}{ PR status } \\
\hline Positive & 80 & 50 & 30 & \multirow[t]{2}{*}{0.404} \\
\hline Negative & 70 & 47 & 23 & \\
\hline \multicolumn{5}{|l|}{ HER2 status } \\
\hline $0 / 1+$ & 113 & 74 & 39 & \multirow[t]{3}{*}{0.726} \\
\hline $2+$ & 26 & 17 & 9 & \\
\hline $3+$ & 11 & 6 & 3 & \\
\hline
\end{tabular}

$E R$ estrogen receptor, $P R$ progesterone receptor, Her 2 human epidermal growth factor receptor 2

$* p<0.05, * * p<0.01$

transduction regardless of the physiological or pathological condition [13]. It has been shown that high expression of GNAS is tightly associated with distal metastasis in breast cancer patients, but whether this phenomenon in fact occurs requires further confirmation. Knockdown of GNAS suppressed wound closure at $48 \mathrm{~h}$ to $54.4 \%$ and $69.7 \%$, respectively, in siControl-treated MDA-MB-231 and MCF-7 cells ( $p<0.05$, Fig. 4a, c), indicating that GNAS was important for breast cancer cell migration.

Moreover, consistent with the clinical analysis and wound healing results, knockdown of GNAS reduced to almost half the number of migrated and invaded cells (209 \pm 13 vs $108 \pm 7$ and $102 \pm 6$ vs $52 \pm 7$ migrated cells; $221 \pm 14$ vs $114 \pm 11$, $116 \pm 4$ vs $56 \pm 7$ invaded cells, respectively, in MDA-MB-231 and MCF-7) (Fig. 4b, d).

\section{GNAS promotes breast cancer cell proliferation and invasion though activation of the PI3K/AKT signaling pathway}

According to our results, GNAS accelerates breast cancer cell proliferation and migration, but the underlying molecular mechanism remains unknown. To gain further insight into the molecular events responsible for this phenomenon, we examined key proteins in signaling pathways that are highly related to GNAS. In siGNAS-treated cells, protein kinase A (PKA), a critical downstream effector of cyclic AMP signaling, was dramatically reduced, which further confirmed the siRNA efficiency (Fig. 5a, b). The PI3K/ AKT signaling pathway is indispensable for cell growth, proliferation, survival, etc., and increasing evidence has documented that an interaction may exist between PI3K/ AKT and cyclic AMP signaling pathway [14-16]. We noticed that although the total AKT level was not altered, the $\mathrm{p} 85 \alpha$ subunit of PI3K and p-AKT was significantly reduced after siGNAS treatment, indicating that the function of GNAS in breast cancer might be partly dependent on the PI3K/AKT signaling pathway.

In addition, it has been well documented that epithelial mesenchymal transition (EMT) is a critical process for cell migration and invasion, as well as scilicet cancer cell metastasis [17]. Given the correlation between high GNAS expression and the presence of distal tumor metastases in breast cancer patients, we examined several key proteins associated with the EMT process. As a result, the expression of E-cadherin was increased 2.08-fold in siGNAStreated cells, indicating an impairment of cell migration and invasion. Although both snail1 and snail2 (slug) are upstream transcription factors of E-cadherin, only the expression of snail1 was reduced by $60 \%$ in siGNAStreated cells (Fig. 5a, b).

To further investigate whether GNAS regulates cell proliferation and EMT through the PI3K/AKT signaling pathway, we selectively inhibited the PI3K/AKT signaling pathway by treating MDA-MB-231 cell with the pan-class I PI3K inhibitor BKM120 [18, 19]. As a result, p-AKT was severely reduced, and the alteration of E-cadherin and snail 1 was consistent with that in siGNAS-treated cells (Fig. 5c). Additionally, the proliferation of MDA-MB-231 cells was dramatically reduced, as assessed by the EdU and colony formation assay (Fig. 5d, e). Moreover, the migration and invasion abilities were also impaired according to the transwell assay results (Fig. 5f). Although the expression of GNAS was unchanged, the inhibition of PI3K/ AKT signaling pathway mimicked the phenotype caused by GNAS silencing, which suggested that GNAS was an upstream factor in the PI3K/AKT signaling pathway. 
Table 2 Univariate and multivariate analysis of different prognostic variables of GNAS with overall survival

\begin{tabular}{|c|c|c|c|c|c|c|c|}
\hline \multirow[t]{2}{*}{ Variables } & \multirow[t]{2}{*}{$n$} & \multicolumn{3}{|c|}{ Univariate analysis } & \multicolumn{3}{|c|}{ Multivariate analysis model } \\
\hline & & HR & $95 \% \mathrm{CI}$ & $p$ value & HR & $95 \% \mathrm{CI}$ & $p$ value \\
\hline WHO grade & & 1.695 & $0.306-1.694$ & 0.821 & N.A & & \\
\hline I & 52 & & & & & & \\
\hline II & 44 & & & & & & \\
\hline III & 54 & & & & & & \\
\hline Tumor size $(\mathrm{cm})$ & & 1.394 & $1.309-2.896$ & 0.137 & N.A & & \\
\hline$<5$ & 74 & & & & & & \\
\hline$\geq 5$ & 72 & & & & & & \\
\hline Nodal status & & 1.068 & $0.758-1.905$ & 0.856 & N.A & & \\
\hline N0-1 & 73 & & & & & & \\
\hline $\mathrm{N} 2-3$ & 77 & & & & & & \\
\hline Distal metastasis & & 1.048 & $0.886-1.934$ & 0.042 & 1.287 & $1.268-3.694$ & $0.015^{*}$ \\
\hline Presence & 70 & & & & & & \\
\hline Absence & 80 & & & & & & \\
\hline Clinical status & & 1.896 & $1.509-2.638$ & 0.038 & 1.736 & $1.868-3.288$ & $0.016^{*}$ \\
\hline I-II & 63 & & & & & & \\
\hline III-IV & 87 & & & & & & \\
\hline ER status & & 1.903 & $0.693-1.766$ & 0.803 & N.A & & \\
\hline Positive & 78 & & & & & & \\
\hline Negative & 72 & & & & & & \\
\hline PR status & & 1.796 & $1.083-2.733$ & 0.963 & N.A & & \\
\hline Positive & 80 & & & & & & \\
\hline Negative & 70 & & & & & & \\
\hline HER2 status & & 1.086 & $1.239-2.703$ & 0.563 & N.A & & \\
\hline $0 / 1+$ & 113 & & & & & & \\
\hline $2+$ & 26 & & & & & & \\
\hline $3+$ & 11 & & & & & & \\
\hline GNAS expression & & 1.126 & $1.086-2.163$ & 0.007 & 1.763 & $1.424-3.809$ & $0.021 *$ \\
\hline High & 97 & & & & & & \\
\hline Low & 53 & & & & & & \\
\hline
\end{tabular}

$H R$ hazard rate, $C I$ confidence interval, $E R$ estrogen receptor, $P R$ progesterone receptor, Her 2 human epidermal growth factor receptor 2

$* p<0.05$

\section{Knockdown of GNAS inhibits tumor growth and EMT in vivo}

To investigate the role of GNAS in tumor growth and metastasis in vivo, an MDA-MB-231 subcutaneous tumor transplantation model was established in nude mice. The tumor volume was measured weekly. Although all the mice vaccinated with MDA-MB-231 cells possessed tumors, the tumor volume in the siGNAS-treated group was significantly reduced, indicating that knockdown of GNAS dramatically delayed tumor growth (Fig. 6a-c). This phenomenon may be partly due to the lower proliferation rate of the siGNAStreated group, as measured by Ki-67 staining (Fig. 6d). To further analyze the EMT status of the newly generated tumor cells, an immunoblotting assay was carried out, and the results were similar to the in vitro situation with an increase in E-cadherin and decrease in Snaill in siGNAS-treated tumors (Fig. 6e, f). Collectively, silencing of GNAS severely affected tumor growth and EMT in vivo.

\section{Discussion}

G protein, as one of the most important signal transducer, plays a pivotal role in pathophysiological conditions, and it has been well documented that its coupled receptors (GPCRs) participate in nearly all kinds of tumorigenesis, including breast cancer [20, 21]. GNAS, the stimulatory subunit of $\mathrm{G}$ protein, has been reported to be related to development, cell proliferation and metabolism, among others, with 
Fig. 2 Silencing of GNAS impairs cell viability and proliferation in MDA-MB-231 cells. The relative expression of GNAS in breast cancer cell lines (a); silencing of GNAS with specific siGNAS in MDAMB-231 cells (b); CCK8 assay measuring cell viability after transfection with siGNAS and siControl (c); the number of cells with newly synthesized DNA was dramatically reduced after siGNAS treatment, as measured by the EdU incorporation assay, scale bar $=25 \mu \mathrm{m}$ (d)
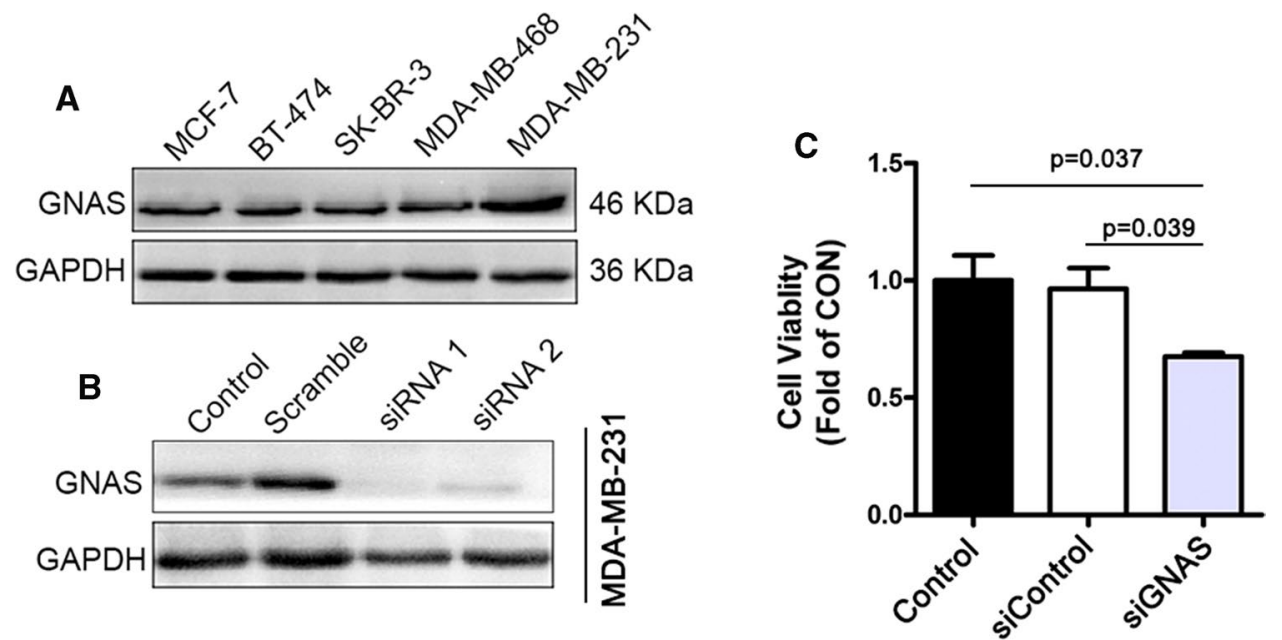
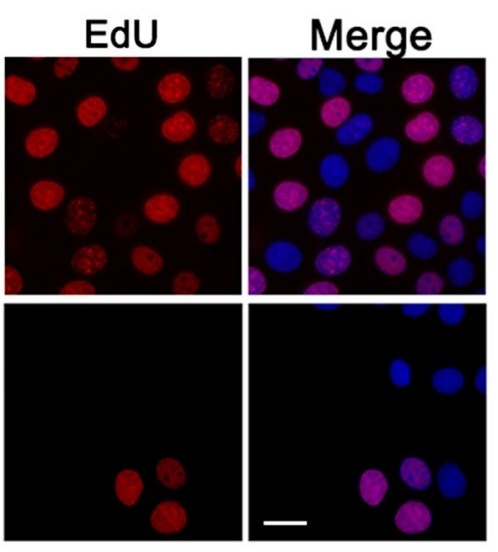

an imprinted expression pattern [22]. Recent studies have demonstrated that mutations that cause GNAS dysfunction are related to various cancers characterized by aberrant cell proliferation, such as pituitary cancer, pancreatic cancer, lung cancer, and intraductal papillary mucinous neoplasms [23-25]. However, the underlying molecular mechanism is poorly understood.

Although several groups have also identified the GNAS mutation in a very small portion of breast cancer patients and 20q amplified breast cancer cell lines [26, 27], the correlation of the expression of GNAS with breast cancer remains unknown. Our current study demonstrated, for the first time, that rather than activating mutation, the expression level of GNAS is associated with breast cancer. Our data demonstrated not only that more than half of the included breast cancer patients had high expression of GNAS, but also that high GNAS expression was significantly associated with an enhanced proliferative ability and metastasis. Moreover, patients with high GNAS expression exhibited poor survival.
Infinite proliferation and metastasis abilities are two major features of cancer cells, which result from numerous signaling pathway disorders. The PI3K/AKT signaling pathway has been well documented and highlighted in numerous studies as the master regulator of carcinogenesis [28]. In the present study, we found an interaction between the PI3K/ AKT and cAMP signaling pathways, and GNAS acted as an upstream effector of the PI3K/AKT signaling pathway, which was able to activate this pathway by increasing $\mathrm{p} 85 \alpha$ and p-AKT expression. Although GPCRs have been reported to activate the PI3K/AKT signaling pathway via insulin receptor substrate 1 (IRS1) or Ras [29, 30], we determined that high expression of GNAS, rather than GPCRs, could result in PI3K/AKT signaling activation.

The relationship between $\mathrm{G}$ proteins and PI3K activity is very complicated, and Robert $\mathrm{T}$ et al. emphasized that PI3K activity is controlled by the $\mathrm{G} \beta \gamma$ subunits of $\mathrm{G}$ proteins [5]. However, increasing evidence has shown that the core downstream effector of GNAS protein kinase A (PKA) plays a central role in the regulation of the PI3K/ 

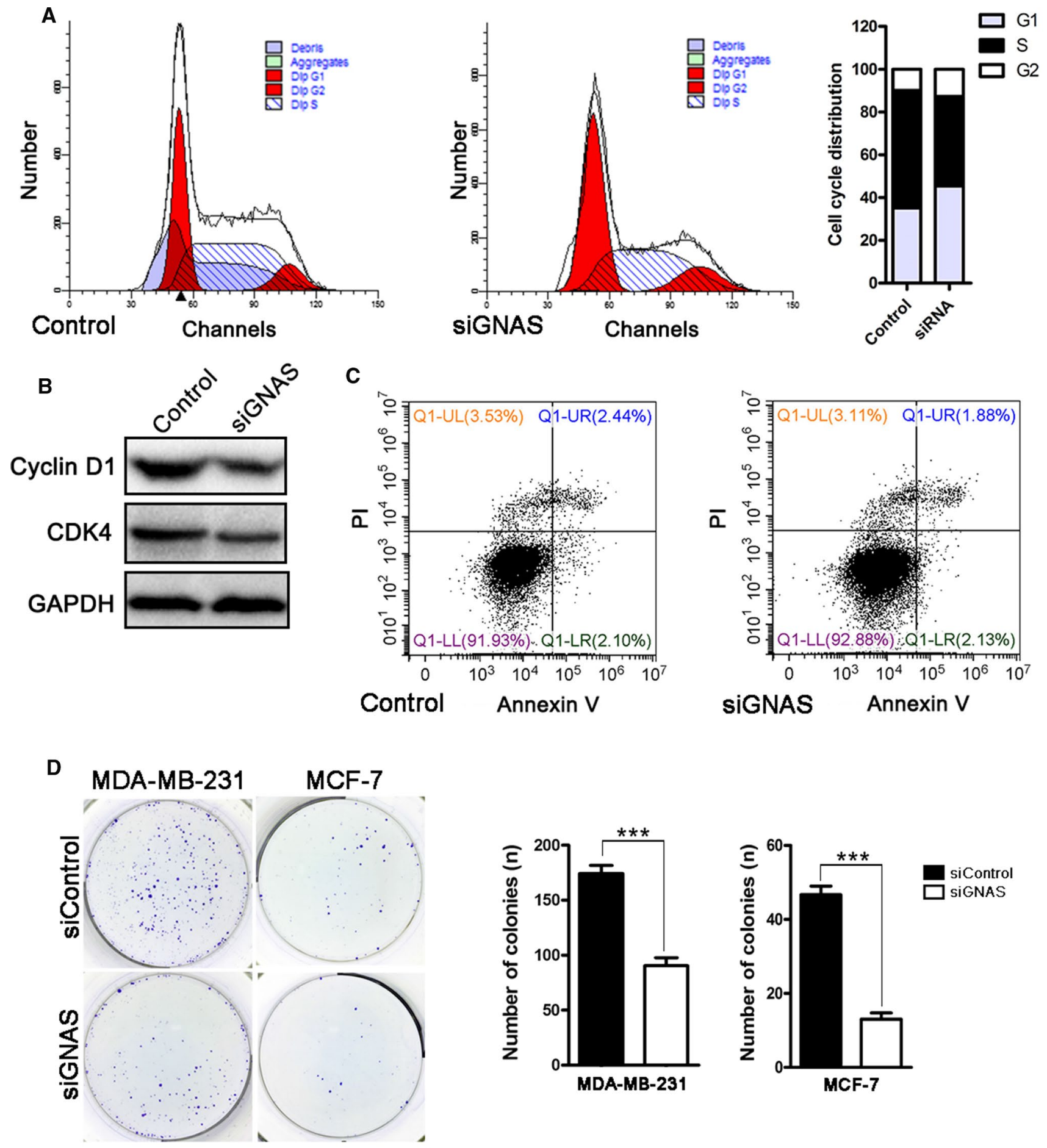

Fig. 3 GNAS regulates the cell cycle distribution and colony formation ability in breast cancer cells. The transition of the G1/S checkpoint is impaired in GNAS knockdown MDA-MB-231 cells (a); the expression of Cyclin D1 and CDK4 is reduced in GNAS insufficient MDA-MB-231 cells (b); no obvious difference was observed in cell

apoptosis between siGNAS and siControl-treated MDA-MB-231 cells (c); the colony formation ability was weakened after GNAS knockdown both in MDA-MB-231 and MCF-7 cells, $p=0.008$ and 0.0004, respectively (d)

AKT signaling pathway in ovarian granulosa cells, which is independent of the $G \beta \gamma$ subunits [31, 32]. Consistent with their results, we also demonstrated that the PI3K/AKT

signaling pathway was activated by the GNAS/PKA axis, thus providing more information related to the interaction between $\mathrm{G}$ proteins and the PI3K/AKT signaling pathway. 

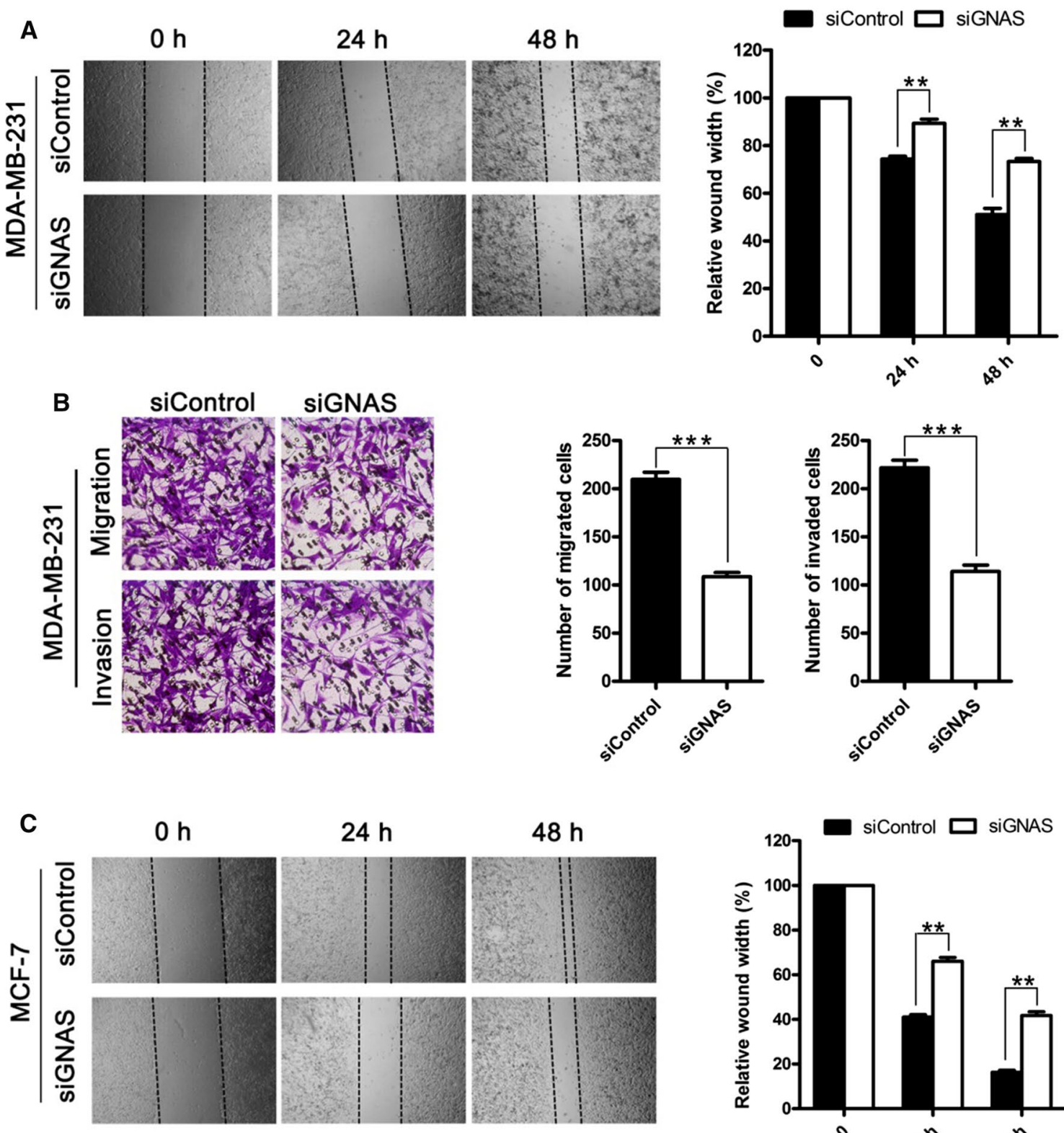

$48 \mathrm{~h}$
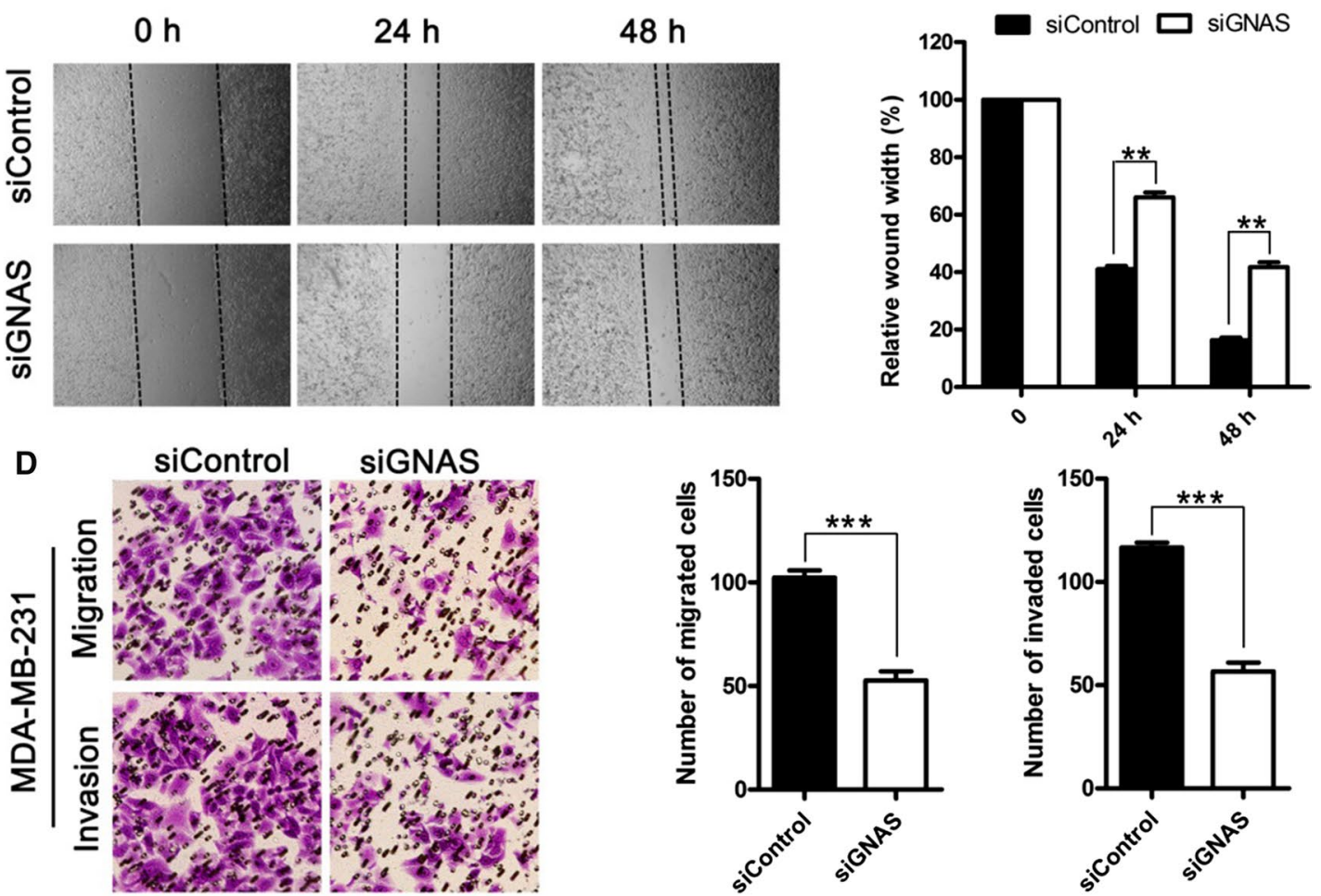

Fig. 4 The wound healing assay was carried out to evaluate cell movement alteration by siGNAS at $24 \mathrm{~h}$ and $48 \mathrm{~h}$ after treatment in MDA-MB-231 (a) and MCF-7 (c), respectively. The Matrigel inva- 

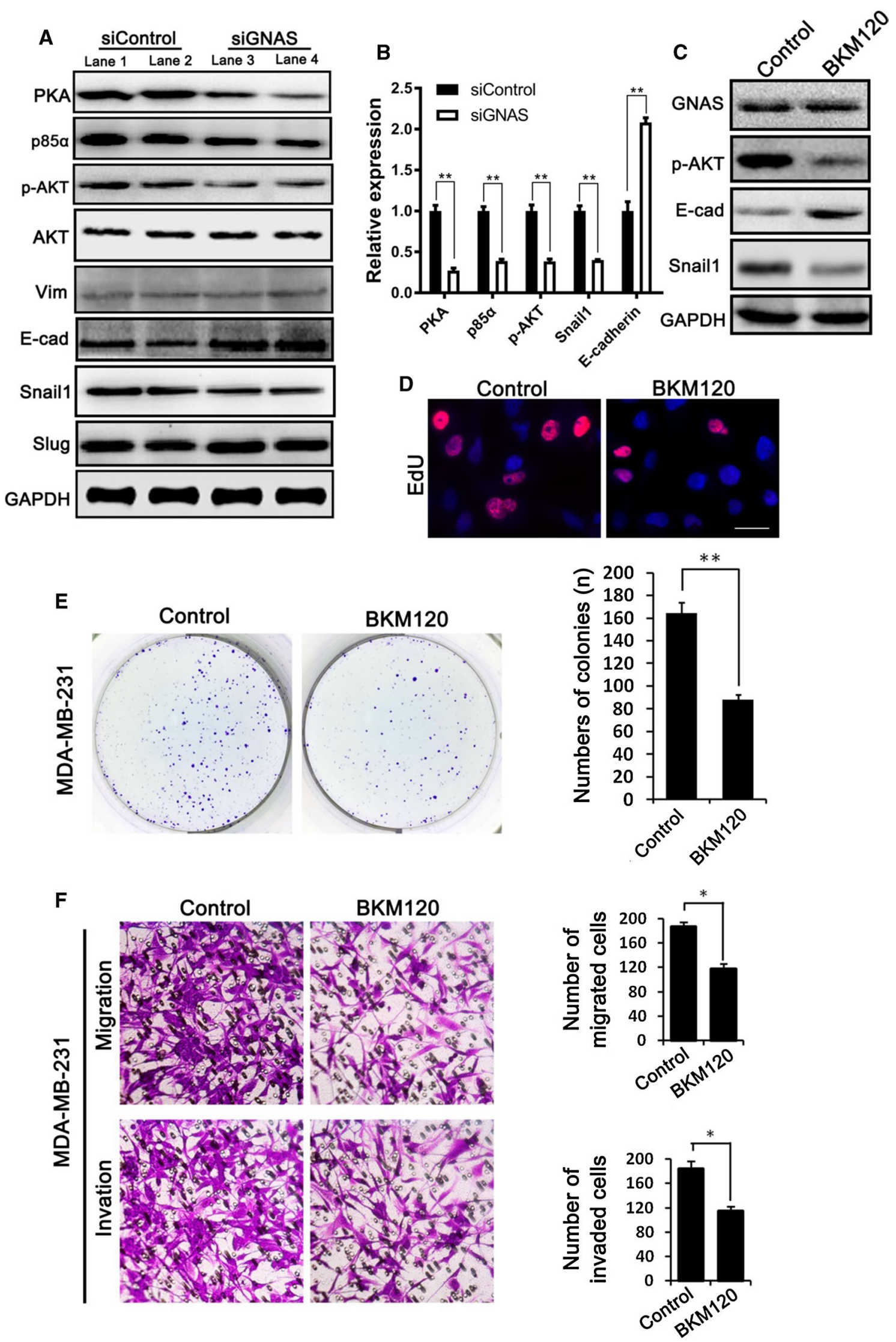
4Fig. 5 The decrease in the PI3K/AKT/Snail1/E-cadherin axis by the siGNAS and PI3K specific inhibitor BKM can mimic the cell proliferation and invasion phenotype analyzed by EdU incorporation and the Matrigel invasion assay in the MDA-MB-231 cell line. The expression of $\mathrm{p} 85 \alpha, \mathrm{p}$-AKT and snaill declined, while E-cadherin exerted the opposite trend with elevated expression, in GNAS insufficient MDA-MB-231 cells (a, b); blockade of PI3Ks with BKM120 resulted in a similar expression pattern of the key proteins (c) and reduced cell proliferation (d) and colony formation ability (e), together with the decreased mobility and invasion ability (f), scale bar $=25 \mu \mathrm{m}$

Additionally, the different subunits of $\mathrm{G}$ proteins may crosstalk in different situations.
E-cadherin, which is frequently used as a marker for the EMT process, was elevated in siGNAS-treated MDAMB-231 cells, together with a decrease in its transcription factor snail 1 rather than slug, which indicated that EMT was inhibited by GNAS silencing. Moreover, blockade of PI3Ks by the specific inhibitor BKM120 resulted in a similar phenotype. Finally, we further confirmed the function of GNAS in cell proliferation and metastasis in vivo using a subcutaneous tumor transplantation model.

In summary, our study revealed that high GNAS expression in breast cancer is significantly associated with tumor growth, metastasis, and poor survival in breast cancer patients. GNAS promotes breast cancer cell proliferation and metastasis through the PKA/PI3K/AKT/Snail1/E-cadherin
Fig. 6 Ablation of GNAS decreased tumor growth and the expression of key EMT markers in vivo. The tumor size was dramatically reduced in siGNAS-treated MDA-MB-231 cells, scale bar $=1 \mathrm{~cm}(\mathbf{a}-\mathbf{c})$, as compared to the control group tumor; GNAS insufficient MDA-MB-231 cell formed tumors showed a lower rate of proliferation, scale bar $=100 \mu \mathrm{m}$ (d); the expression of p-AKT and snaill was reduced, while the expression of E-cadherin was elevated, in siGNAS-treated tumors (e); quantification of the expression of p-AKT, E-cadherin and Snail1 (f)

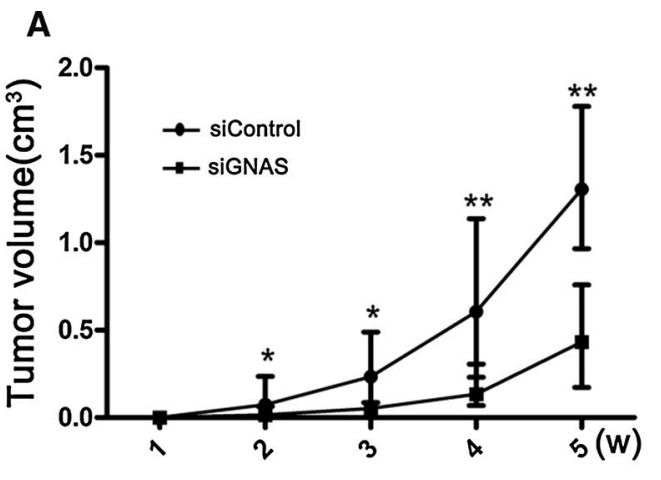

B

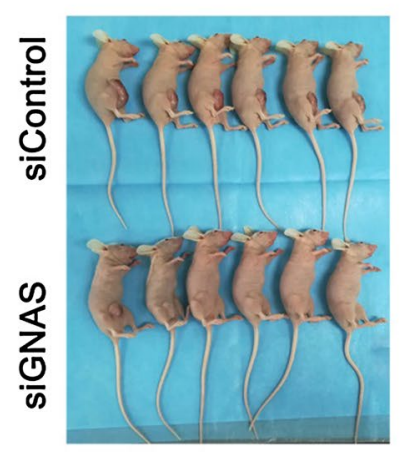

C
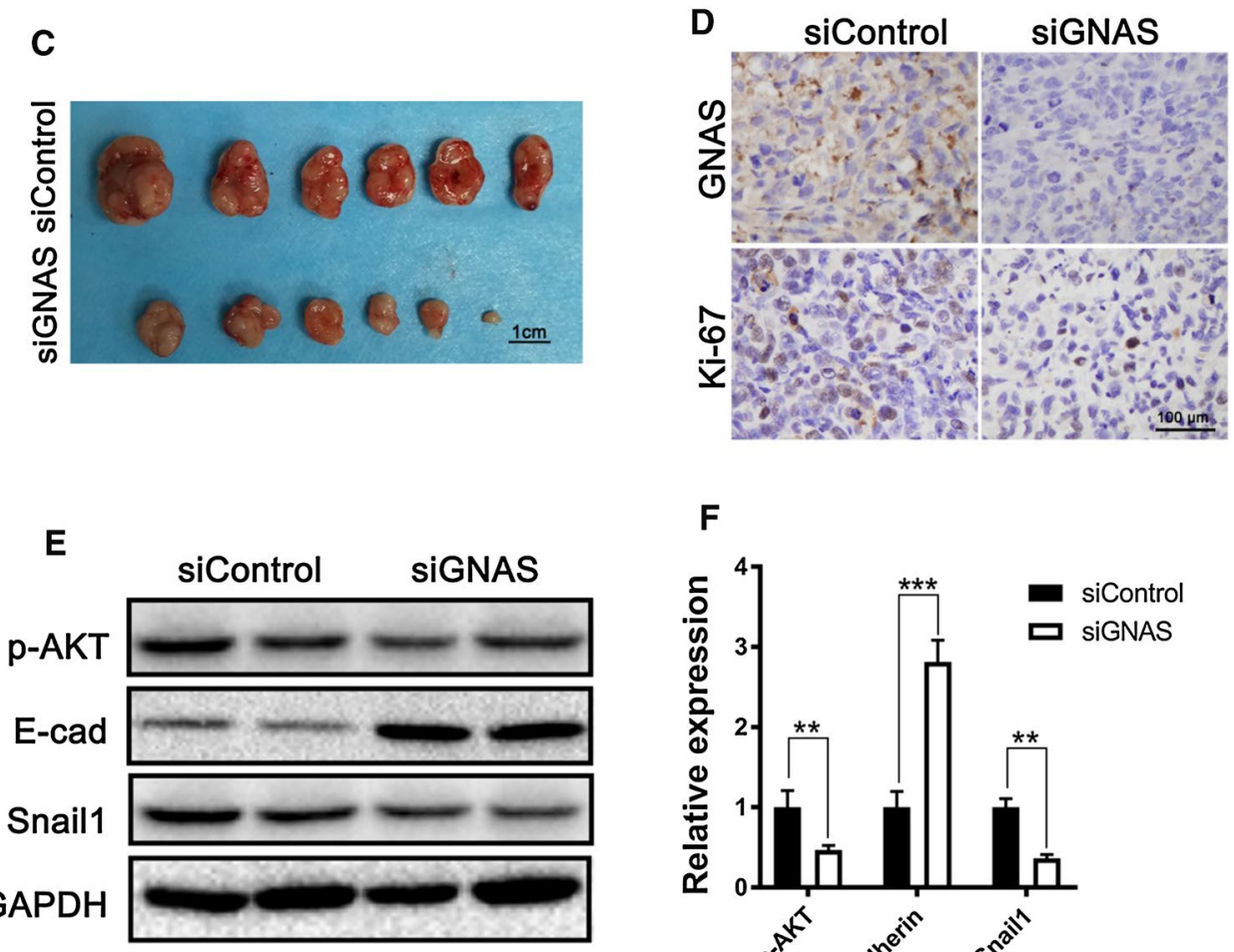

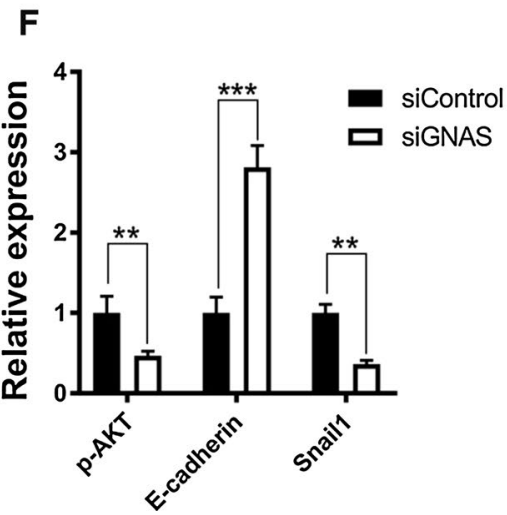


axis, and GNAS can serve as a potential prognostic indicator and novel therapeutic target of breast cancer.

Funding This study was funded by the Key Research Projects on Application Foundation of Sichuan Science and Technology Department (No. 2017JY0030) and Yibin Health and Family Planning Commission.

\section{Compliance with ethical standards}

Conflict of interest The authors declare no potential conflicts of interest in the study.

Research involving human participants and animals All procedures performed in studies involving human participants and animals were in accordance with the ethical standards of the institutional and/or national research committee and with the 1964 Helsinki declaration and its later amendments or comparable ethical standards.

Informed consent Informed consent was obtained from all individual participants included in the study.

Open Access This article is distributed under the terms of the Creative Commons Attribution 4.0 International License (http://creativeco mmons.org/licenses/by/4.0/), which permits unrestricted use, distribution, and reproduction in any medium, provided you give appropriate credit to the original author(s) and the source, provide a link to the Creative Commons license, and indicate if changes were made.

\section{References}

1. Jayaraman S, Doucet M, Lau WM, Kominsky SL. CITED2 modulates breast cancer metastatic ability through effects on IKK $\alpha$. Mol Cancer Res. 2016;14:730.

2. Lu J, Steeg PS, Price JE, Krishnamurthy S, Mani SA, Reuben J, et al. Breast cancer metastasis: challenges and opportunities. Cancer Res. 2009;69:4951.

3. Feigin ME. Harnessing the genome for characterization of G-protein coupled receptors in cancer pathogenesis. FEBS J. 2013;280:4729-38.

4. Lappano R, Maggiolini M. G protein-coupled receptors: novel targets for drug discovery in cancer. Nat Rev Drug Discov. 2011;10:47.

5. Dorsam RT, Gutkind JS. G-protein-coupled receptors and cancer. Nat Rev Cancer. 2007;7:79-94.

6. O’Hayre M, Vázquez-Prado J, Kufareva I, Stawiski EW, Handel TM, Seshagiri S, et al. The emerging mutational landscape of G-proteins and G-protein coupled receptors in cancer. Nat Rev Cancer. 2013;13:412.

7. Lu C, Xia J, Zhou Y, Lu X, Zhang L, Gou M, et al. Loss of Gs $\alpha$ impairs liver regeneration through a defect in the crosstalk between cAMP and growth factor signaling. J Hepatol. 2016;64:342-51.

8. Das R, Esposito V, Abu-Abed M, Anand GS, Taylor SS, Melacini G, et al. cAMP activation of PKA defines an ancient signaling mechanism. Proc Natl Acad Sci USA. 2007;104:93-8.
9. Beristain AG, Molyneux SD, Joshi PA, Pomroy NC, Di Grappa MA, Chang MC, et al. PKA signaling drives mammary tumorigenesis through Src. Oncogene. 2015;34:1160-73.

10. Bhat RR, Yadav P, Sahay D, Bhargava DK, Creighton CJ, Yazdanfard S, et al. GPCRs profiling and identification of GPR110 as a potential new target in HER2+ breast cancer. Breast Cancer Research and Treatment. 2018;170(2):279-92.

11. Rao A, Herr DR. G protein-coupled receptor GPR 19 regulates E-cadherin expression and invasion of breast cancer cells. Biochim Biophys Acta (BBA) Mol Cell Res. 2017;186:1318.

12. Liang S, Chen Z, Jiang G, Zhou Y, Liu Q, Su Q, et al. Activation of GPER suppresses migration and angiogenesis of triple negative breast cancer via inhibition of NF-kB/IL-6 signals. Cancer Lett. 2017;386:12.

13. Chen M, Gavrilova O, Zhao WQ, Nguyen A, Lorenzo J, Shen $\mathrm{L}$, et al. Increased glucose tolerance and reduced adiposity in the absence of fasting hypoglycemia in mice with liver-specific Gsalpha deficiency. J Clin Investig. 2005;115:3217-27.

14. Manning BD, Cantley LC. AKT/PKB signaling: navigating downstream. Cell. 2007;129:1261-74.

15. Khalil BD, Hsueh C, Cao Y, Abi Saab WF, Wang Y, Condeelis JS, et al. GPCR signaling mediates tumor metastasis via PI3K $\beta$. Cancer Res. 2016;76:2944-53.

16. Donini CF, Di ZE, Zuchegna C, Di Domenico M, D'Inzeo S, Nicolussi A, et al. The p $85 \alpha$ regulatory subunit of PI3K mediates cAMP-PKA and retinoic acid biological effects on MCF7 cell growth and migration. Int J Oncol. 2012;40:1627-35.

17. Bill R, Christofori G. The relevance of EMT in breast cancer metastasis: correlation or causality? FEBS Lett. 2015;589:1577-87.

18. Ni J, Ramkissoon SH, Xie S, Goel S, Stover DG, Guo H, et al. Combination inhibition of PI3K and mTORC1 yields durable remissions in orthotopic patient-derived xenografts of HER2-positive breast cancer brain metastases. Nat Med. 2016;22:723-6.

19. Liu H, Murphy CJ, Karreth FA, Emdal KB, White FM, Elemento $\mathrm{O}$, et al. Identifying and targeting sporadic oncogenic genetic aberrations in mouse models of triple negative breast cancer. Cancer Discov. 2018;8:354-69.

20. Syrovatkina V, Alegre KO, Dey R, Huang XY. Regulation, signaling and physiological functions of G-proteins. J Mol Biol. 2016;428:3850-68.

21. Ghosh P. The untapped potential of tyrosine-based G protein signaling. Pharmacol Res. 2016;105:99-107.

22. Peters J, Holmes R, Monk D, Beechey CV, Moore GE, Williamson CM. Imprinting control within the compact Gnas locus. Cytogenet Genome Res. 2006;113:194-201.

23. Mantovani G, Lania AG, Spada A. GNAS imprinting and pituitary tumors. Mol Cell Endocrinol. 2010;326:15-8.

24. Ideno N, Ohtsuka T, Matsunaga T, Kimura H, Watanabe Y, Tamura K, et al. Clinical significance of GNAS mutation in intraductal papillary mucinous neoplasm of the pancreas with concomitant pancreatic ductal adenocarcinoma. Pancreas. 2015;44:311.

25. Ritterhouse LL, Vivero M, Mino-Kenudson M, Sholl LM, Iafrate AJ, Nardi V, et al. GNAS mutations in primary mucinous and nonmucinous lung adenocarcinomas. Mod Pathol. 2017;30:1720-7.

26. Volckmar AL, Leichsenring J, Flechtenmacher C, Pfarr N, Siebolts $\mathrm{U}$, Kirchner M, et al. Tubular, lactating, and ductal adenomas are devoid of MED12 Exon2 mutations, and ductal adenomas show recurrent mutations in GNAS and the PI3K-AKT pathway. Genes Chromosom Cancer. 2016;56:11-7.

27. Garcia-Murillas I, Sharpe R, Pearson A, Campbell J, Natrajan R, Ashworth A, et al. An siRNA screen identifies the GNAS locus as a driver in 20q amplified breast cancer. Oncogene. 2013;33:2478-86. 
28. Martini M, De Santis MC, Braccini L, Gulluni F, Hirsch E. PI3K/ AKT signaling pathway and cancer: an updated review. Ann Med. 2014;46:372-83.

29. Law NC, White MF, Hunzickerdunn ME. G protein-coupled receptors (GPCRs) that signal via protein kinase A (PKA) cross-talk at insulin receptor substrate 1 (IRS1) to activate the phosphatidylinositol 3-kinase (PI3K)/AKT pathway. J Biol Chem. 2016;291:27160-9.

30. Suire S, Lécureuil C, Anderson KE, Damoulakis G, Niewczas I, Davidson K, et al. GPCR activation of Ras and PI3Kc in neutrophils depends on PLCb2/b3 and the RasGEF RasGRP4. EMBO J. 2012;31:3118-29.

31. Law NC, White MF, Hunzickerdunn ME. G protein-coupled receptors (GPCRs) that signal via protein kinase A (PKA) cross-talk at insulin receptor substrate 1 (IRS1) to activate the phosphatidylinositol 3-kinase (PI3K)/AKT pathway. J Biol Chem. 2016;291(53):27160-9.

32. Hunzickerdunn ME, Lopezbiladeau B, Law NC, Daniel WC, Carr DW, Maizels ET. PKA and GAB2 play central roles in the FSH signaling pathway to PI3K and AKT in ovarian granulosa cells. Proc Natl Acad Sci U S A. 2012;109(44):17742-3.

Publisher's Note Springer Nature remains neutral with regard to jurisdictional claims in published maps and institutional affiliations. 